Quaderni di Geografia Cahiers de Gécongaty rataphie

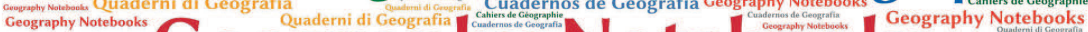
Cuadernos de Geografia Geography Notebooks $U 201000$ Cahiers de Géographi Cahiers de Géographie cahiers de Géographie Cuadernos de Geografía Cahiers de Géographie Cahiers de Géographie Cuadernos de Geografía Geography Notebooks

\author{
$4(2021)$ \\ 1 \\ Teatro di suoni. \\ Spazi acustici teatrali e territoriali
}

A cura di

Martino Mocchi, Lorena Rocca, Demis Quadri and Carlotta Sillano

EDITORIAL

Teatro di suoni per l'attaccamento ai luoghi. Uno sguardo geografico 11

Lorena Rocca

Per un teatro di suoni. Riflessioni su possibili dimensioni sonore nelle 23 creazioni site-specific di physical theatre

Demis Quadri

INTRODUCTION

Teatro di suoni. Spazi acustici teatrali e territoriali

Demis Quadri e Lorena Rocca

SPECIAL Issue

I suoni di Mantova come strumenti di interpretazione del paesaggio.

Tra turismo sostenibile ed educazione al patrimonio culturale

Valeria Pecorelli, Franca Zuccoli, Alessandra De Nicola, Enrico Squarcina

Il paesaggio sonoro campano tra contemporaneità e nuove forme

di progettualità turistica

Germana Citarella 
La narrazione spettacolarizzata del paesaggio sonoro.

Da Giuseppe Chiari a Philip K. Dick e oltre

Francesco Michi

Musica di paesaggi sonori. Enunciazione, risignificazione, comunicazione

Carlotta Sillano

Camminare per ascoltare. Partiture invisibili del territorio abitato

Elisabetta Senesi

Il paesaggio sonoro in relazione. Suono, movimento e immagini per stimolare complessità percettiva Angela Calia

Groove Fields. Understanding the Dance Floor from an Art-Based Research Perspective

Sebastian Mattbias

Il silenzio come esperienza trasformativa. L'importanza del silenzio nella meditazione e in ambito professionale

Sebastiano Caroni

Progettare il silenzio. Una lettura acustica dell'ex villaggio sanatoriale 125 Morelli a Sondalo

Martino Mocchi

Voicing One's Will. Theatre as Audio-Visual Hypotyposis of the Poetic

Michael Groneberg

Music and Clowning in Europe, 20th-21st centuries

Anna Stoll Knecht

Il paesaggio sonoro nella composizione musicale. Un percorso bibliografico

Stefano Alessandretti

\#exploreART: il labirinto di A. Pomodoro e i bambini. Un progetto di fruizione condivisa con percorsi sensoriali partecipati Alessandra De Nicola, Franca Zuccoli 


\section{OTHER EXPLORATIONS}

Il rumore lontano. Intervista a Lorena Rocca

a cura di Martino Mocchi

Re Cervo. Intervista a Antonella Astolfi

a cura di Krizia Bonaudo e Demis Quadri

Centovalli-Centoricordi. Intervista a Oliviero Giovannoni a cura di Krizia Bonaudo e Demis Quadri

Alla ricerca di un metodo: Open Space Technology 



\title{
I suoni di Mantova come strumenti di interpretazione del paesaggio. Tra turismo sostenibile ed educazione al patrimonio culturale
}

\author{
Valeria Pecorelli ${ }^{1}$, Franca Zuccoli ${ }^{1}$, Alessandra De Nicola ${ }^{2}$, \\ Enrico Squarcina ${ }^{1}$ \\ ${ }^{1}$ Dipartimento di Scienze Umane per la Formazione "Riccardo Massa", \\ Università degli Studi di Milano Bicocca \\ ${ }^{2}$ BIPAC Interdepartmental research Centre for Cultural and Artistic \\ Heritage, Università degli Studi di Milano Bicocca
}

DoI: https://doi.org/10.7358/gn-2021-001-peco

\begin{abstract}
This article presents an educational experience in schools held by academics in the UNESCO site of Mantova and Sabbioneta. It is the result of a wider, interdisciplinary study - sponsored by MOBARTECH, an Italian research project - aimed at the co-construction of knowledge to foster understanding and interpretation of the cultural landscape from the point of view of the local community. The project led to the creation of a toolkit for citizens and tourists in which sound was the protagonist of several activities. Here we critically present insights and reflections on heritage, landscape and the sound in education.
\end{abstract}

Keywords: Unesco; cultural heritage; citizens; schools; education.

Parole chiave: Unesco; patrimonio culturale; cittadini; scuole; educazione.

\section{INTRODUZIONE ${ }^{1}$}

${ }^{1}$ Il contributo è frutto di un progetto comune, ma i singoli paragrafi sono stati redatti individualmente come di seguito esplicitato: paragrafo 1 - Squarcina; paragrafo 2 - Zuccoli; paragrafo 3 - De Nicola; paragrafo 4 - Pecorelli. Le conclusioni sono a cura di tutti gli autori. 
Il presente lavoro racconta un'esperienza formativa, nell'ambito di un percorso di alternanza scuola-lavoro, volta a migliorare la fruizione del paesaggio e del patrimonio del sito Unesco di Mantova e Sabbioneta. Si tratta di un progetto di ricerca italiano denominato MOBARTECH. Una piattaforma tecnologica per lo studio e la valorizzazione di beni storico-artistici. Caso studio n. 2: il sito UNESCO di Mantova e Sabbioneta realizzato con fondi di Regione Lombardia, cofinanziato con il FESR per gli investimenti in favore della crescita e dell'occupazione, i cui obiettivi generali sono la ricerca industriale, lo sviluppo sperimentale, l'innovazione dei processi e delle organizzazioni.

Adottando un approccio multidisciplinare, il gruppo di ricerca coinvolto si è posto come obiettivo la co-costruzione della conoscenza al fine di incoraggiare la creazione di nuovi processi creativi volti a comprendere e a interpretare il paesaggio culturale dal punto di vista della comunità locale, con una ricaduta operativa sia per i residenti, sia per i turisti.

A partire dai principi ispiratori espressi dalla Convenzione di Faro $(2005)^{2}$, si è ritenuto significativo prendere in esame il caso studio qui riportato considerando la sua identità istituzionale di sito Unesco, tipologia che potrebbe essere interpretata come un'etichettatura di "patrimonio dall'alto". Nello specifico, si tratta di un progetto di ricerca-azione concluso nel 2021, il cui obiettivo finale è stato la creazione di un kit di fruizione del patrimonio culturale adatto al pubblico inteso nel modo più eterogeneo possibile. A tal fine, accanto a un lavoro di indagine intrapreso grazie alla somministrazione di questionari costruiti a partire dai riferimenti trovati nella letteratura scientifica, uniti alla realizzazione di interviste mirate a cittadini e a testimoni privilegiati, è stato svolto un percorso di alternanza scuola lavoro il cui risultato conclusivo è stato la creazione e la sperimentazione di un prototipo del kit che partisse dall'esperienza formativa compiuta. Attualmente, con il kit sperimentale testato e modificato raccogliendo le osservazioni dei fruitori, si ipotizza che questa modalità operativa, basata su una reale progettazione partecipata di cittadini e studenti, possa fornire alcune idee e alcuni spunti critici per lo sviluppo di una concettualizzazione a partire dalle pratiche condivise sperimentate relative patrimonio, al di là delle tradizionali narrazioni culturali e accademiche.

${ }^{2}$ La Convenzione chiama le popolazioni a svolgere un ruolo attivo nel riconoscimento dei valori dell'eredità culturale e invita gli stati a promuovere un processo di valorizzazione partecipativo, fondato sulla sinergia tra istituzioni pubbliche, cittadini privati e associazioni. 


\section{LA COSTRUZIONE DEL KIT: BREVI RIFLESSIONI METODOLOGICHE}

Il kit progettato attraverso questa ricerca nasce da una stretta collaborazione con le istituzioni delle due città coinvolte, Mantova e Sabbioneta, unite all'ufficio Unesco, che da sempre lavora per potenziare il coinvolgimento degli studenti di tutti gli ordini e i gradi scolastici, oltre che in sinergia con le associazioni culturali presenti sul territorio. Insieme a loro si sono individuate alcune scuole con cui poter realizzare il lavoro. Grazie alla collaborazione avuta con alcuni studenti dell'Istituto tecnico a indirizzo turistico Pitentino di Mantova, si è potuto riflettere concretamente sul rapporto imprescindibile tra patrimonio, paesaggio e storie locali.

Durante il percorso formativo i ragazzi hanno osservato e sperimentato le diverse metodologie di valorizzazione del patrimonio culturale, le fonti da cui attingere per poter avere un approccio critico e personale relativo all'interpretazione dei luoghi, avendo la possibilità inoltre di utilizzare in diretta alcuni strumenti di fruizione. Dopo diverse esplorazioni è stato prodotto un prototipo di kit esperienziale, inteso come strumento per potenziare le attività di turismo partecipativo relative al sito Unesco. I prototipi sono stati costruiti con l'intento di coinvolgere, nel prossimo futuro, diverse tipologie di pubblico (famiglie, turisti stranieri, utenti delle città, studenti di ogni età). Il kit contiene strumenti dal forte taglio empirico quali mappe, cartoline con attività ludiche, artistiche, sonore, corporee (Dewey 1951), volti a coinvolgere i sensi per interpretare i luoghi e i patrimoni attraverso il proprio punto di vista, limitando quanto più possibile l'intermediazione della guida o di un formatore. L'approccio multisensoriale comporta uno sguardo sul patrimonio di tipo fenomenologico, più legato alla percezione che al sapere nozionistico.

L'obiettivo della ricerca consisteva nel coniugare l'esperienza di tipo sensoriale con informazioni, tali da trasformarsi in conoscenze scientificamente validate. Il kit prodotto industrialmente dalla casa editrice Terre di Mezzo è il frutto di un processo partecipato di costante proposta e selezione delle attività, scandito da tre fasi corrispondenti ad altrettanti prototipi, costantemente verificati con i cittadini. Grazie a questo percorso, sono stati messi a punto strumenti per migliorare l'osservazione e favorire un'esplorazione attiva (frame, filtri colorati, specchi, cartoline illustranti il passato e immagini di dettaglio). Un altro strumento è il quaderno delle attività, al cui interno si trovano proposte che coinvolgono pratiche quali l'autobiografismo, l'educazione corporea, l'attenzione agli elementi vegetali e architettonici, fino a esercizi di esplorazione sonora.

A questi ausili si accompagna anche una carta di Mantova e di Sab- 
bioneta in cui i punti di riferimento e i siti sono stati scelti in base alle prospettive dei residenti senza seguire i percorsi tradizionali del turismo di massa. Si è tornati così a far rivivere antiche storie locali, dando voce alle ricette di cibi tradizionali, oltre che alla sapienza dei procedimenti artigianali delle botteghe storiche, e ai luoghi reputati personalmente significativi. Questo strumento, attraverso la tecnologia $Q R$ code, ha permesso di mettere a disposizione del visitatore molti dei contenuti digitali di tipo sonoro e visivo prodotti o raccolti.

Come sostiene Robertson (2016) il patrimonio culturale riguarda le persone e, nello specifico, quello che i cittadini compiono ogni giorno. Allo stesso modo, il paesaggio culturale non è solo l'espressione di una singola prospettiva, ma una costruzione complessa e multiforme, carica di potere simbolico. Abitare o frequentare quotidianamente un luogo, che nel caso di Mantova e Sabbioneta è riconosciuto di eccezionale valore universale, tale da essere inserito nella lista del Patrimonio Mondiale, non significa necessariamente conoscerlo. Come affermano Lashua e Baker (2014) nelle città e nei siti dove sono già previste etichette istituzionali, come nel caso qui presentato, che viene celebrato come gemma nascosta del Rinascimento italiano, il patrimonio può essere dato per scontato, con il rischio reale di non essere neanche osservato, per il fatto stesso di esser percepito come dato immobile, cristallizzato nel tempo. Spesso ciò che diventa abitudinario perde nella nostra percezione il suo valore, quasi fossimo anestetizzati rispetto al suo effetto (Zuccoli and De Nicola 2018). A partire da questo dato di fatto, più volte sperimentato dall'amministrazione di Mantova, un'équipe multidisciplinare, composta da pedagogisti, didatti, psicologi, geografi, afferente al Dipartimento di Scienze umane dell'Università di Milano-Bicocca, coinvolta nel progetto Mobartech ha intrapreso il suo lavoro di ricerca.

Ricercatori, borsisti e docenti hanno realizzato un continuo confronto, motivato dal fatto che il paesaggio è un costrutto complesso e sfaccettato che implica diversi punti di vista e differenti prospettive e che non si può chiudere in un'unica e statica definizione, ma che ha bisogno del dialogo tra una molteplicità di discipline. Se, a titolo esemplificativo, per i geografi appartenenti a un determinato paradigma di riferimento il paesaggio è uno sguardo (Raffestein 2005), per uno storico dell'arte può essere assimilabile alla pittura che ci insegna cosa vedere in natura (Gombrich 1977), dal punto di vista pedagogico può essere la straordinaria occasione per fare esperienze significative a partire dai sensi (Rousseau 2017). In questa prospettiva pedagogica, come afferma Castiglioni (2012), l'educazione al paesaggio può collocarsi entro il più ampio campo 
dell'educazione allo sviluppo sostenibile, quale percorso volto a costruire un rapporto equilibrato e duraturo con l'ambiente in cui si vive.

La riflessione condivisa alla luce dei dati qualitativi raccolti in situ conferma quanto definisce la Convenzione Europea sul Paesaggio (2000) e cioè che il paesaggio è risultato per noi quasi un'invenzione dei cittadini, un patrimonio da tutelare e valorizzare, parte fondante delle identità. Questo aspetto così importante e valoriale, pare come qualcosa a cui non si è più abituati, oggetto di esperienze extra-ordinarie come ad esempio i luoghi "esotici" di alcune vacanze sognate.

\section{IL SUONO: UNO STRUMENTO ALTERNATIVO DI INTERPRETAZIONE DEL PATRIMONIO}

Legato a questo aspetto in bilico tra una vita quotidiana da cittadino, assorbita dalle occupazioni, e una prospettiva dell'uso degli stessi luoghi da parte dei turisti, pare di cogliere la sovrapposizione di traiettorie con orizzonti diversi. Nell'era del tanto declamato "turismo sostenibile", si assiste a un'ulteriore differenziazione, accanto a una crescente domanda di turismo nelle città d'arte, che sta producendo una tipologia turistica propria di una massificazione di stampo capitalista, troviamo il proliferare di percorsi di turismo alternativo considerati anche questi in qualche modo colpevoli di sostenere la gentrificazione (Weaver 2014), il degrado e l'aumento di traffico (Borghi and Celata 2009).

Il progetto proposto ha avuto come obiettivo prioritario la creazione di un modello sostenibile per la fruizione di un patrimonio più condiviso e partecipato al di là delle narrazioni culturali tradizionali, proprio perché si è avvalso della collaborazione degli abitanti e in particolare dei cittadini più giovani. Tuttavia, senza una prima alfabetizzazione volta a generare consapevolezza del patrimonio culturale, non era possibile creare un atteggiamento partecipativo sostenibile nei confronti della cultura locale. Il primo motivo del nostro agire è stato riportare, allora, il significato di patrimonio a un ambiente quotidiano sulla base di una richiesta dell'Amministrazione locale mantovana dell'ufficio Unesco che ha rilevato nel corso della sua attività l'assenza della consapevolezza da parte degli abitanti del valore del luogo in cui vivono. A partire da questa criticità, è nata la proposta di nuovi strumenti di interpretazione.

Al fine di non "museificare" il paesaggio urbano preso in esame e tenendo in considerazione la definizione Unesco (2003) di patrimonio im- 
materiale, che include come componente essenziale il suono ${ }^{3}$, si è deciso in corso d'opera di integrare e sviluppare la dimensione sonora all'interno dei processi di elaborazione del kit di fruizione della città. Il paesaggio sonoro risulta essere poco indagato dagli studi legati al turismo, tuttavia, laddove la dimensione sonora viene tenuta in considerazione, la qualità dell'offerta turistica migliora, come dimostrano recenti studi (Lui et al 2018). Sulla scorta di esperienze di esplorazione del territorio urbano precedenti, come il progetto didattico Sentire il paesaggio promosso dall'Ufficio Unesco di Mantova e Sabbioneta nell'A.S. 2014/2015, coordinato da Cinzia Lanfredi Sofia e realizzato dal musicoterapista Simone Rizzardi, o il caso della cartolina sonora inviata dalla Piazza Mantegna di Mantova alla piattaforma Écouter le monde ${ }^{4}$, ci si è resi conto del valore della componente sonora in un percorso di scoperta del territorio. Un ascolto attento e concentrato nell'ambiente esplorato, in relazione con la dimensione storica, culturale, sociale e naturale, favorisce una riscoperta del territorio personale. L'esplorazione sonora, in questo senso, facilita una maggiore consapevolezza della realtà, dell'intimo senso dei luoghi e delle identità locali. Come ricorda Martino Mocchi, argomentando le tesi di Peter Zumthor sul concetto di 'atmosfera', «il ricordo spesso si costruisce in assenza di elementi riconoscibili e dominanti sullo sfondo in modo assoluto, attingendo invece a una serie di esperienze tra loro disomogenee e frammentarie, che hanno a che fare con il clima del momento, con le condizioni atmosferiche, i suoni percepiti, con la particolare luce che si riflette sui colori, con gli odori del luogo, ecc. È appunto questo complesso di sensazioni e di disposizioni soggettive, emotive che definisce l'“atmosfera" di un luogo» $(2017,74)$.

Le attività proposte nel kit hanno fra i loro obiettivi quello di facilitare la costruzione dell'atmosfera, dal punto di vista personale, delle due città, per questo l'approccio attraverso il soundscape è stato fondamentale. Era questo, infatti, uno degli elementi inizialmente non contemplato dalle altre azioni di scoperta del territorio, che permetteva di riscoprire i luoghi, a partire da un punto di osservazione poco approfondito. A seconda dei casi il suono evoca situazioni, memorie di esperienze precedentemente vissute o più semplicemente permette di ampliare il bagaglio di conoscenze legate a un luogo. Si è lavorato in particolare sui suoni di tipo "events", vale a dire, quella tipologia fortemente legata a uno spazio fisico, che supera i saperi di tipo musicale. A partire da questi si è mirato

\footnotetext{
${ }^{3}$ https://unesdoc.unesco.org/ark:/48223/pf0000259172.locale=fr

${ }^{4}$ https://www.ecouterlemonde.net/fr/item/elm/ELM_SS_Mantoue_bus_00
} 
a ricostruire le realtà esplorate attraverso sensazioni elementari.

L'aspetto sonoro diviene così lo strumento per creare una rinnovata geografia dei luoghi che passa anche attraverso l'identificazione di ambienti acustici (Pocock 1989). A partire da questa prospettiva si può affermare che con questa, seppur iniziale sperimentazione, si sia andati a costruire dei nuovi primi paesaggi sonori condivisi, motore di una progressione più approfondita.

\section{Percezioni sonore a confronto}

Nella prima fase legata all'introduzione del suono, in questo progetto, si è partiti da tre domande stimolo. La richiesta fatta agli studenti dell'alternanza scuola-lavoro è stata quella di ragionare sulla città di Mantova, individuando rispetto alla tematica sonora, tre tipologie così contraddistinte: il suono più poetico, quello più inaspettato e quello più lontano nel tempo. Nonostante la maggioranza dei ragazzi inizialmente apparisse spiazzata e una po' restia nel trattare la tematica, una parte di loro ha riconosciuto alla città di Mantova la presenza di "rumori" e non di "suoni".

Questa affermazione ci permette di collegarci alla categorizzazione di Murray Schafer $(1985 ; 1998)$, che definisce un paesaggio sonoro lo- $f$ in cui il traffico, le auto, i suoni conosciuti come tipicamente urbani soffocano tutto il patrimonio sonoro restante. Si vuole qui riportare anche la testimonianza di uno studente, il quale ha tenuto a precisare come nella sua città non sembrasse possibile cogliere suoni riconoscibili, per il fatto stesso che l'unica impronta sonora comprensibile fosse riconducibile esclusivamente al sovrapporsi caotico di clacson delle automobili e motori degli autobus. Come afferma Stefano Malatesta (2006) gli oggetti sonori che contribuiscono all'inquinamento acustico sono parte irrinunciabile del paesaggio cittadino in cui viviamo. In questa prospettiva Mantova sembrerebbe avere una proprietà acustica lontana dall'idealizzazione di una immagine turistica di cittadina rinascimentale. Un altro studente, però, riusciva con il suo intervento a sottolineare, in alcuni punti della città, la presenza di alcuni suoni propri della natura: "il cinguettio degli uccelli, i rumori delle acque dei laghi, le foglie degli alberi che costeggiano la passeggiata lungo il Mincio" sottolineando una percezione duale, quasi schizofrenica della città: "come se Mantova fosse una città divisa; una della natura e una del quotidiano”.

In una seconda fase della progettazione abbiamo sviluppato ulteriormente le attività legate all'ascolto del paesaggio introducendo nell'équipe 
di ricerca anche una musicista e un'esperta di musicologia, Federica Furlani, al fine di introdurre in modo più mirato e attento il senso dell'udito come uno strumento formale per la conoscenza e l'interpretazione del patrimonio del sito Unesco preso in oggetto. Grazie all'apporto di un approccio sonoro più competente sono state elaborate nuove proposte con lo scopo non solo di ampliare le attività previste per il kit, volte a creare una maggiore consapevolezza all'ascolto del paesaggio sonoro, ma anche per sviluppare una maggiore capacità di saper descrivere, verbalizzare una percezione sonora. Ciò è stato sperimentato attraverso una prima attività laboratoriale, una passeggiata sonora, soundwalk (Springgay and Truman 2018), dall'edificio scolastico al parco di Palazzo Te, con i ragazzi della scuola secondaria di secondo grado dell'Istituto Superiore Bonomi Mazzolara. L'esperienza è stata pensata come ricerca dell'attivazione del senso dell'udito riflettendo sulle capacità di memoria sonora e di abilità nel descrivere a parole il mondo sonoro. La restituzione del racconto sonoro ha saputo evidenziare gli elementi che danno identità sonora al luogo. La maggior parte dei ragazzi ha affermato di aver sentito più suoni di quelli a cui normalmente presta attenzione: "un tosaerba ronzante", "il suono cupo delle macchine", "il suono ritmico e continuo dei passi", "il suono in movimento delle biciclette", "il suono sbriciolante dei sassolini della ghiaia".

Se per Mantova le stimolazioni si sono concentrate sul cercare un'identità sonora per una città che presenta un ambiente acustico molto denso, per Sabbioneta si è cercato un'altra prospettiva ovvero abitare col suono spazi vuoti e silenziosi. Qui è stato applicato il concetto di partitura grafica "per suonare il patrimonio" sul disegno della pianta del Teatro all'Antica, che rappresenta uno dei simboli dell'unicità architettonica e culturale locale. I ragazzi in questo caso hanno ideato alcune sonorizzazioni che potessero rispecchiare il disegno di uno spazio interno e le hanno eseguite con la loro voce e il loro corpo come brevi composizioni musicali. Alla fine delle sperimentazioni sono state scelte le attività che meglio potevano veicolare l'interpretazione del senso del luogo e l'unicità del patrimonio anche in termini sonori.

\section{ConClusioni}

A partire da queste semplici sperimentazioni prodotte per giungere alla costruzione di un kit rivolto ai cittadini e ai turisti di Mantova e Sabbioneta, possiamo affermare che, benché il suono e il paesaggio sonoro delle due città non fosse stato preso in considerazione in modo significativo 
fin dall'inizio del progetto, durante lo svolgimento del lavoro con i ragazzi e con gli adulti questo aspetto abbia assunto un ruolo sempre più rilevante. Tra gli aspetti più significativi del suono, infatti, si può notare una funzione strumentale che permette di creare forme coinvolgenti per approcciarsi in maniera attiva al patrimonio culturale. Tuttavia, anche a partire dall'esperienza brevemente descritta possiamo notare come il suono sia il grande assente nell'immaginario collettivo nonostante Dell'Agnese e Tabusi ricordino che «i suoni possono caratterizzare il contesto, (...) diventando talora un aspetto capace di segnare gli spazi geografici anche in senso identitario» $(2016,6)$. Si tratta di un lavoro di scoperta, difficilmente immediato, anche nel nostro caso i ragazzi dopo uno sforzo di concentrazione hanno saputo identificare e associare delle tipologie sonore assimilabili ai contesti locali. Questo dimostra in primis la necessità di indagare ulteriormente questo strumento interpretativo al fine di aumentare una proposta fruitiva che sia in primo luogo consapevole. Inoltre, come sottolineato da Minidio (2005) al fine di avere una realtà interpretata nella sua totale essenza, è necessario sviluppare una percezione che coinvolga tutti i sensi partendo dal suono che caratterizza il cronotopo (Rocca 2017) e testimonia l'unicità di un dato territorio.

Come affermato da più autori e sperimentato nelle varie ricerche di ambito geografico, le idee di luogo hanno origine attraverso il fertile connubio tra vissuto personale e esperienza, «ma anche mediante la ricezione di stimoli culturali, tra i quali l'arte ha un ruolo assai importante» (Dell'Agnese and Tabusi 2016, 5). Tali stimoli, che a loro volta possono influenzare la percezione diretta, non si limitano all'aspetto visuale, ma sono veicolati anche mediante gli altri sensi, permettendo di coinvolgere il corpo completamente, e non dimenticando di valorizzare un aspetto fondante che è quello legato al suono e all'udito.

\section{RIFERIMENTI BIBLIOGRAFICI}

Borghi, R. and F. Celata. 2009. Turismo critico. Immaginari geografici, performace e paradossi sulle rotte del turismo alternativo. Milano: Unicopli.

Calanchi, A., ed. 2015. Il suono percepito, il suono raccontato. Paesaggi sonori in prospettiva multidisciplinare. Teramo: Galaad Edizioni.

Castiglioni, B. 2012. "Il paesaggio come strumento educativo". Education y futuro: revista de investigaciòn aplicada y experiencias educativas 27: 51-65. 
Consiglio d'Europa. 2005. Convenzione di Faro, CETS n. 199. Faro.

Consiglio d'Europa. 2000. Convenzione Europea del Paesaggio. CETS n. 176. Firenze.

De Nicola, A. e F. Zuccoli, eds. 2016. Paesaggi culturali. Nuove forme di valorizzazione del patrimonio: dalla ricerca all'azione condivisa. Santarcangelo di Romagna: Maggioli.

Dell'Agnese, E. and M. Tabusi, a cura di. 2016. La musica come geografia: suoni, luoghi, territori. Roma: Società geografica italiana.

Dewey, J. 1951. Arte come esperienza. Firenze: La Nuova Italia.

Gombrich, E. 1977. La Storia dell'arte raccontata da E. H. Gombrich. Torino: Einaudi.

Lashua, B. D. and S. Baker. 2014. "Cinema beneath the stars, heritage from below". Who needs experts: 133-145.

Lui, A., X. L. Wang, F. Liu, C. Yao and Z. Deng. 2018. "Soundscapes and its influence on tourist satisfaction”. The Service Industries Journal 38: 3-4.

Malatesta, S. 2006. "Educare al paesaggio sonoro". AST 2: 25-27.

Minidio, A. 2005. I suoni del mondo, studi geografici sul paesaggio sonoro. Milano: Guerini.

Mocchi, M. 2017. "Il progetto multisensoriale". In Tecnologie e processi per il progetto del paesaggio. Reti e modelli distrettuali, a cura di Daniele Fanzini, 71-89. Santarcangelo di Romagna: Maggioli.

Pocock, D. 1989. "Sound and the Geographer". Geography: 193-200.

Raffestin, C. 2005. Dalla nostalgia del territorio, al desiderio di paesaggio. Elementi di una teoria di paesaggio. Firenze: Alinea.

Robertson, I., ed. 2016. Heritage from below. London: Routledge.

Rocca, L. 2017. "Attraverso paesaggi sonori: un workshop per la preservazione e valorizzazione del suono”. Métis: história \& cultura 16 (32): 43-59.

Rousseau, J.J. 2017. Emilio o dell'educazione. Roma: Edizioni Studium.

Schafer, M. R. 1985. Il paesaggio sonoro. Milano: Ricordi.

Schafer, M. R. 1998. Educazione al suono. 100 esercizi per ascoltare e produrre il suono. Milano: Ricordi.

Springgay, S. and S. E. Truman. 2018. Walking Methodologies in a More-than-Human World: WalkingLab. Oxon-New York: Routledge.

Weaver, D. B. 2014. "Asymmetrical dialectics of sustainable tourism: Toward enlightened mass tourism”. Journal of Travel Research 53 (2): 131-140.

Zuccoli, F. e A. De Nicola. 2018. "Patrimoni e ricerche tra materialità e tecnologia". In Ambienti digitali per l'educazione all'arte e al patrimonio, a cura di Alessandro Luigini and Chiara Panciroli, 294-306. Milano: Franco Angeli. 